\title{
Indication of metronomic chemotherapy for metastatic breast cancer: Clinical outcomes and responsive subtypes
}

\author{
KEIICHI KONTANI ${ }^{1}$, SHIN-ICHIRO HASHIMOTO ${ }^{1}$, CHISA MURAZAWA $^{1}$, SHOKO NORIMURA ${ }^{2}$, \\ HIROAKI TANAKA $^{3}$, MASAHIRO OHTANI ${ }^{4}$, NAOMI FUJIWARA-HONJO ${ }^{5}$, MANABU DATE ${ }^{6}$, \\ KOJI TERAMOTO $^{7}$, HITOSHI HOUCHI ${ }^{3}$ and HIROYASU YOKOMISE ${ }^{1}$
}

\author{
${ }^{1}$ Department of Thoracic, Breast and Endocrine Surgery, Kagawa University Faculty of Medicine, Miki, Kagawa 761-0793; \\ ${ }^{2}$ Department of Surgery, Japanese Red Cross Hospital, Takamatsu, Kagawa 760-0017; ${ }^{3}$ Department of Pharmacy, \\ Kagawa University Hospital, Miki, Kagawa 761-0793; ${ }^{4}$ Kagawa Health Service Association, Health Care Center, \\ Takamatsu, Kagawa 761-8071; ${ }^{5}$ Department of Radiology, Osaka Neurosurgery Hospital, Takamatsu, \\ Kagawa 761-8083; ${ }^{6}$ Department of Surgery, Date Hospital, Takamatsu, Kagawa 760-0076; \\ ${ }^{7}$ Department of Surgery, Shiga University of Medical Science, Otsu, Shiga 520-2191, Japan
}

Received January 20, 2016; Accepted March 21, 2016

DOI: $10.3892 / \mathrm{mco} .2016 .841$

\begin{abstract}
The survival of patients with metastatic breast cancer (MBC) has not improved, despite recent advances in therapeutic strategies. This is mainly due to the fact that cytotoxic agents cannot be administered over a long period, even if they exhibit favorable activity, due to treatment-related side effects or acquisition of tumor resistance to the administered agents. Thus, the development of therapeutic strategies that may be used over a long time period is required to improve survival. We assessed the availability and clinical outcomes of metronomic chemotherapy, which is defined as continuous or frequent treatment with low doses of cytotoxic drugs. A total of 80 patients with $\mathrm{MBC}$ received chemotherapy in the metastatic setting, and the clinicopathological factors and clinical outcomes were retrospectively compared between 52 patients who received metronomic regimens and 28 patients who received other cytotoxic regimens. As regards clinical outcomes, the median time-to-treatment failure (TTF) and overall survival (OS) were significantly longer in the metronomic
\end{abstract}

Correspondence to: Dr Keiichi Kontani, Department of Thoracic, Breast and Endocrine Surgery, Kagawa University Faculty of Medicine, 1750-1 Ikenobe, Miki, Kagawa 761-0793, Japan E-mail: konbat@med.kagawa-u.ac.jp

Abbreviations: $\mathrm{CBR}$, clinical benefit rate; $\mathrm{CR}$, complete response; DCR, disease control rate; DFI, disease-free interval; HER2, human epidermal growth factor receptor 2; $\mathrm{MBC}$, metastatic breast cancer; ORR, overall response rate; OS, overall survival; $\mathrm{PD}$, progressive disease; $\mathrm{PR}$, partial response; SD, stable disease; TTF, time-to-treatment failure; TN, triple-negative; TTP, time-to-progression

Key words: metastatic breast cancer, metronomic chemotherapy, luminal type, time-to-treatment failure, overall survival, triple-negative cancer group compared with those in the non-metronomic group (TTF, 15 vs. 4 months, $\mathrm{P}=0.0001$; and $\mathrm{OS}, 53$ vs. 28 months $\mathrm{P}=0.0012$, respectively). In the metronomic group, none of the 18 patients who responded to the regimen had triple-negative (TN) cancer (17 had luminal-type tumors and 1 had a human epidermal factor receptor 2-type tumor). Furthermore, TTF and OS were significantly longer in patients with non-TN cancer compared with those in patients with $\mathrm{TN}$ cancer in the metronomic group (TTF, 16 vs. 7 months, $\mathrm{P}=0.0014$; and OS, 108 vs. 20 months, $\mathrm{P}=0.000007$, respectively). The proportion of patients who experienced treatment-related adverse events was significantly lower in the metronomic group compared with that in the non-metronomic group ( 36.5 vs. $61.5 \%$, respectively; $\mathrm{P}=0.038$ ). In conclusion, metronomic chemotherapy is a viable option for luminal-type $\mathrm{MBC}$ in terms of effectiveness and minimal toxicity, regardless of metastatic sites or prior treatment. However, an alternative treatment is required for $\mathrm{TN}$ cancer.

\section{Introduction}

Breast cancer has a relatively favorable prognosis compared with the prognosis of other cancers, such as lung, colon, ovarian and pancreatic cancers. The survival of patients with primary breast cancer has significantly improved due to recent advances in therapeutic strategies. However, the treatment of metastatic breast cancer (MBC) has not improved significantly and the outcomes remain unsatisfactory. Although anthracyclines and taxanes are the representative agents used as standard chemotherapy in the adjuvant setting, these agents have also been used in several cases of MBC (1-4) and selecting therapeutic regimens in this setting may be difficult for physicians. In cases of hormone-insensitive tumors, or cases of hormone-sensitive tumors that exhibit resistance to endocrine therapy, cytotoxic agents that have not been previously administered, or taxanes in combination with molecular-targeted agents, are potential candidates (4-9). Recently, taxanes in combination 
with bevacizumab, which targets the tumor vasculature to reduce blood supply to the tumors (7-9), or eribulin, which is an analogue of halichondrin B targeting tubulin in tumor cells, have demonstrated favorable efficacy in improving the prognosis of patients with MBC (10). While a number of these available cytotoxic agents have been shown to exert a suppressive effect on tumor growth, their effects are not maintained for a long period, due to the acquisition of resistance to these agents by the tumor cells, attenuated physical status of the patients or intolerable treatment-related adverse effects. Thus, to prolong the survival of the patients with MBC, alternative therapeutic strategies are required to achieve a long-term response or enable long-term administration of the regimens.

We previously reported that the administration of one therapeutic regimen for $\geq 1$ year was one of the most important factors for long-term survival in the metastatic setting (11). We also demonstrated that more MBC patients who survived for $\geq 60$ months following MBC diagnosis had received metronomic chemotherapy, compared with patients exhibiting shorter survival (12). Metronomic chemotherapy, which is defined as continuous or frequent treatment with low doses of anticancer agents, is commonly used as palliative care in patients who have been heavily pretreated with cytotoxic drugs or who have poor performance status (13). Interestingly, metronomic chemotherapy used for palliation has been reported to result in favorable tumor responses and prolonged survival of the treated patients (14-17). Metronomic chemotherapy may be continued over a long period of time due to its minimal toxicity. However, this strategy has not been investigated in detail in terms of its mechanism of action, outcome and toxicity. The aim of this study, was to compare the clinicopathological characteristics and clinical outcome between patients with MBC who received metronomic chemotherapy and those who received non-metronomic regimens.

\section{Patients and methods}

Patients. Data from 80 patients with advanced or recurrent breast cancer, who were treated with chemotherapeutic regimens at Kagawa University Hospital from February, 2005 to June, 2014, were retrospectively analyzed. To assess the usefulness of metronomic chemotherapy in the metastatic setting, the patients were divided into two groups according to whether they had received metronomic chemotherapy as treatment for $\mathrm{MBC}$. The metronomic group included 52 patients who had received metronomic regimens at least once in the metastatic setting, and the non-metronomic group, which included 28 patients who had not received a metronomic regimen but had received other cytotoxic agents. The metronomic regimens included capecitabine $(1,800 \mathrm{mg} /$ body/day) alone or in combination with cyclophosphamide (100 mg/body/day) administered in cycles of 14 consecutive days repeated at 3-week intervals, or TS-1 (100 mg/body/day) alone or in combination with cyclophosphamide (100 mg/body/day) administered in cycles of 14 consecutive days repeated at 3 -week intervals. For some patients with hormone-sensitive tumors, endocrine therapy consisting of either tamoxifen, letrozole or exemestane, was added to the metronomic regimen at the physician's discretion.
For patients with human epidermal factor receptor 2 (HER2)overexpressing tumors, trastuzumab was added to the metronomic regimen.

The present study was in compliance with the guidelines of the Ethics Committee of Kagawa University Hospital and conformed to the provisions of the Declaration of Helsinki in 1995. All the study patients provided written informed consent.

Evaluation of therapeutic efficacy. Tumor response was assessed by physical examination and computed tomography, magnetic resonance imaging or bone scan, according to the Response Evaluation Criteria in Solid Tumors (18), every 2-3 months during treatment. Complete response (CR) was defined as the absence of evidence of disease, partial response (PR) was defined as a reduction in the product of the two largest perpendicular diameters of the target lesions by $\geq 50 \%$, and progressive disease (PD) was defined as an increase in tumor size by $\geq 25 \%$, or presence of a new lesion. Clinical response that did not meet any of the abovementioned definitions was classified as stable disease (SD). CR and PR were defined as objective response. $\mathrm{CR}, \mathrm{PR}$ and $\mathrm{SD}$ were defined as disease control rate (DCR), and CR, PR and SD observed for $\geq 6$ months were defined as clinical benefit rate (CBR). The clinical outcomes examined in this study included time-to-treatment failure (TTF), defined as the duration from initiation to discontinuation of treatment, time-to-progression (TTP), defined as the duration from initiation of treatment to disease progression or death from any cause, and overall survival (OS), defined as the duration from initiation of treatment to death from any cause. Toxicity was assessed according to the National Cancer Institute Common Toxicity Criteria, version 3.0 (19). For patients who had received $\geq 2$ cytotoxic regimens for $\mathrm{MBC}$, survival time was calculated from the administration of the first metronomic regimen in the metronomic group, or the most effective cytotoxic regimen in the non-metronomic group.

Statistical analysis. The by Mann-Whitney U test or the standard Chi-square test were used for comparisons between the two groups. The effects of baseline characteristics, clinical response or prognostic parameters on the risk of progression or death were assessed using the Kaplan-Meier survival analysis and the log-rank test of significance. A 95\% confidence interval for the median of each variable was computed using the method of Brookmeyer and Crowley. $\mathrm{P}<0.05$ was considered to indicate statistically significant differences; all P-values were two-sided. The SPSS statistical software system, version 23 (SPSS Inc., Tokyo, Japan) was used for all calculations.

\section{Results}

Baseline characteristics of the patients. The median age of the patients was 59 years (range, 32-82 years). Hormone-sensitive and HER2-overexpressing tumors accounted for 59.0 and $21.9 \%$ of the cases, respectively (Table I). When the clinicopathological characteristics were compared between patients who had received metronomic regimens and those who had received a non-metronomic regimen, the median age was 
Table I. Clinicopathological characteristics of patients who received metronomic regimens and those who received other regimens.

\begin{tabular}{|c|c|c|c|c|}
\hline Characteristics & All patients & $\begin{array}{l}\text { Patients receiving } \\
\text { MET }^{\mathrm{b}}\end{array}$ & $\begin{array}{l}\text { Patients not } \\
\text { receiving MET }\end{array}$ & P-value \\
\hline Patient no. & 80 & 52 & 28 & \\
\hline Age, years & $59(32-82)$ & $60(40-81)$ & $51(32-82)$ & 0.0013 \\
\hline Tumor grade & $2(1-3)$ & $2(1-3)$ & $3(1-3)$ & 0.025 \\
\hline DFI, months & $37(3-24)$ & $42(10-241)$ & $37(3-128)$ & 0.201 \\
\hline Disease sites, $\mathrm{n}$ & $2(1-6)$ & $2(1-6)$ & $2(1-6)$ & 0.869 \\
\hline Visceral lesions, $\%$ & 56.2 & 53.8 & 57.7 & 0.75 \\
\hline AT pretreatment, $\%$ & 40.7 & 39.2 & 42.3 & 0.872 \\
\hline Hormone-sensitive tumors, $\%$ & 59 & 63.5 & 50 & 0.258 \\
\hline HER2-overexpressing tumors, $\%$ & 21.9 & 23.4 & 19.3 & 0.743 \\
\hline Triple-negative tumors, $\%$ & 30.8 & 25 & 42.3 & 0.121 \\
\hline Chemotherapy cycles for BC, $\mathrm{n}$ & $4(1-7)$ & $4(1-7)$ & $3(1-5)$ & 0.034 \\
\hline Chemotherapy cycles for MBC, $\mathrm{n}$ & $3(1-7)$ & $3(1-7)$ & $2(1-4)$ & 0.0018 \\
\hline
\end{tabular}

Values are presented as median (range). Bold print indicates statistical significance. MET, metronomic regimen; DFI, disease-free interval; AT, anthracyclines and taxanes; HER2, human epidermal growth factor receptor 2; BC, breast cancer; MBC, metastatic breast cancer.

Table II. Clinical outcomes of patients who received and those who did not receive metronomic regimen.

\begin{tabular}{lcrr}
\hline Outcomes & Patients receiving MET & Patients not receiving MET & P-value \\
\hline Response rate, $\%$ & 36 & 52 & 0.167 \\
Clinical benefit rate, $\%$ & 68 & 53.8 & 0.368 \\
Disease control rate, $\%$ & 74 & 61.5 & 0.346 \\
Median TTF, months & 15 & 4 & $\mathbf{0 . 0 0 0 1}$ \\
Median TTP, months & 20 & 5 & $\mathbf{0 . 0 1 4}$ \\
Median OS, months & 53 & 30 & $\mathbf{0 . 0 0 1 2}$ \\
Median OS from MBC, months & 103 & 30 & $\mathbf{0 . 0 0 0 3}$ \\
\hline
\end{tabular}

MET, metronomic regimen; TTF, time-to-treatment failure; TTP, time-to-progression; OS, overall survival; MBC, metastatic breast cancer.

significantly higher and the tumor grade was significantly lower in the metronomic group compared with those in the non-metronomic group (age, 60 vs. 52 years, $\mathrm{P}=0.0013$; and tumor grade, 2 vs. $3, \mathrm{P}=0.025$, respectively). The number of chemotherapy regimens administered prior to the metronomic regimen, or of other cytotoxic regimens that achieved the best response among regimens that patients had received in the metastatic setting, was higher in the metronomic compared with the non-metronomic group (3 vs. 2, respectively; $\mathrm{P}=0.0018)$. Other baseline characteristics, including disease-free interval (DFI), number of disease sites, hormone receptor and HER2 status of the tumor, prior anthracycline or taxane treatment and the proportion of patients with visceral lesions, did not differ significantly between the two groups.

Efficacy of treatment and clinical outcomes. When clinical outcomes were compared between patients who had received metronomic regimens and those who had received a non-metronomic regimen, overall response rate (ORR), CBR or DCR did not differ between the two groups (Table II). However, the median TTF, TTP and OS were significantly longer in the metronomic group compared with those in the non-metronomic group (TTF, 15 vs. 4 months, $\mathrm{P}=0.0001$; TTP, 20 vs. 5 months, $\mathrm{P}=0.014$; and $\mathrm{OS}, 53$ vs. 28 months, $\mathrm{P}=0.0012$, respectively; Table II and Fig. 1). The OS from the time of MBC diagnosis also appeared to be significantly longer in the metronomic group $(\mathrm{P}=0.0003)$. Therefore, metronomic regimens were associated with a significantly more favorable prognosis in $\mathrm{MBC}$, regardless of the tumor response to treatment.

To identify the factors responsible for the favorable clinical outcome with metronomic regimens, clinicopathological factors and clinical outcomes were compared between patients who responded to metronomic regimens and non-responders. All but one of the patients who responded to metronomic regimens had hormone-sensitive tumors, whereas patients with hormone-sensitive tumors accounted for $<50 \%$ of the patients who failed to respond to this regimen (94.4 vs. 47.1\%, respectively, $\mathrm{P}=0.0008$; Table III). One patient without luminal-type tumor who responded to the metronomic regimen had an HER2-overexpressing tumor. Of note, none of the patients with TN tumors responded to metronomic regimens. Other factors, such as age, tumor 
Table III. Comparison of clinical outcomes of patients achieving CR and those not achieving CR.

\begin{tabular}{lccc}
\hline Outcomes & Responders & Non-responders & P-value \\
\hline Patient no. & 18 & 34 & 0.35 \\
Age, years & $59.5(36-82)$ & $60.5(43-81)$ & 0.36 \\
Tumor grade & $1(1-3)$ & $2(1-3)$ & 0.61 \\
DFI, months & $60(24-172)$ & $32(10-241)$ & 0.328 \\
Disease sites, $n$ & $2(1-5)$ & 52.9 & 0.859 \\
Visceral lesions, $\%$ & 55.5 & 38.2 & 0.667 \\
AT pretreatment, $\%$ & 44.4 & 47.1 & $\mathbf{0 . 0 0 0 8}$ \\
Hormone-sensitive tumors, $\%$ & 94.4 & 18.8 & 0.276 \\
HER2-overexpressing tumors, $\%$ & 33.3 & 38.2 & $\mathbf{0 . 0 0 2 7}$ \\
Triple-negative tumors, $\%$ & 0 & $4.44 \pm 1.97$ & 0.25 \\
Chemotherapy cycles for BC, $\mathrm{n}$ & $3.82 \pm 1.92$ & $3.97 \pm 2.01$ & 0.1 \\
Chemotherapy cycles for MBC, $\mathrm{n}$ & $3.12 \pm 1.64$ & &
\end{tabular}

Values are presented as median (range). Bold print indicates statistical significance. CR, complete response; DFI, disease-free interval; AT, anthracyclines and taxanes; HER2, human epidermal growth factor receptor 2; BC, breast cancer; MBC, metastatic breast cancer.

A

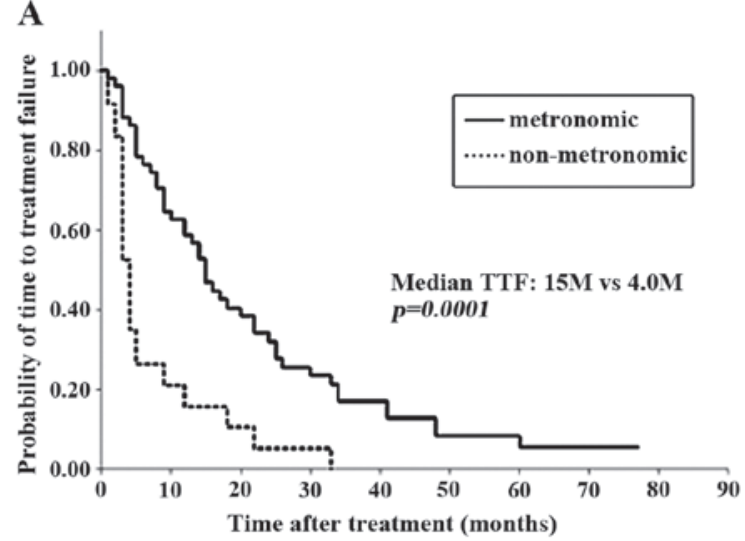

B

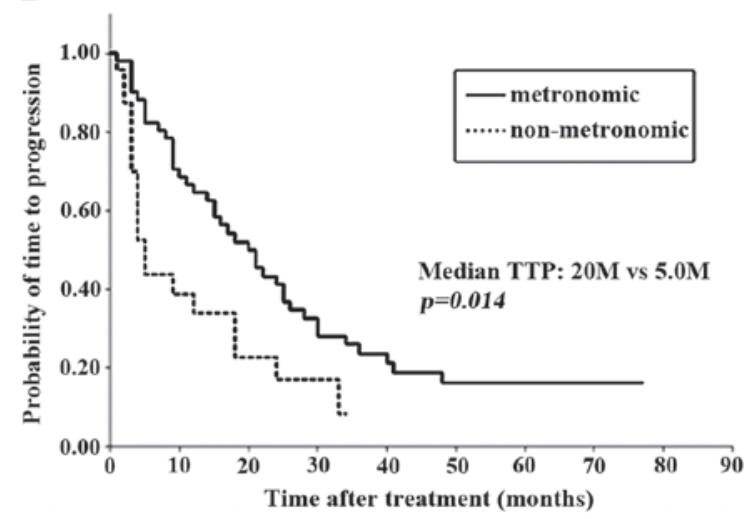

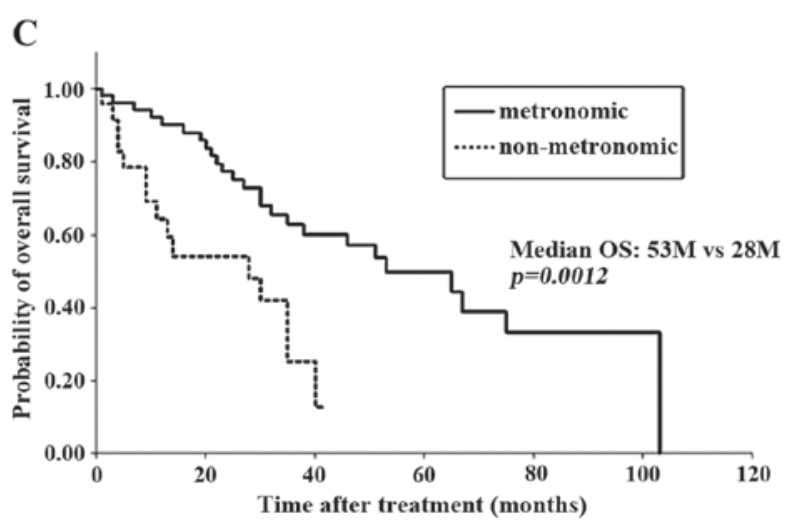

Figure 1. Clinical outcomes of patients who received a metronomic regimen and of those who did not receive this type of regimen. (A) Time-to-treatment failure (TTF); (B) time-to-progression (TTP); and (C) overall survival (OS).

grade, DFI, HER2 status of the tumor and prior treatment, did not differ significantly between the two groups. When comparing clinical outcomes between patients who responded and those who did not respond to a metronomic regimen, all prognostic factors, i.e., TTF, TTP and OS, were significantly longer in responders compared with non-responders (TTF,
33 vs. 9 months, $\mathrm{P}=0.0002$; TTP, 41 vs. 11 months, $\mathrm{P}=0.0023$; and $\mathrm{OS}$, not reached vs. 30 months, $\mathrm{P}=0.00002$, respectively; Table IV). These data suggested that a favorable response to a metronomic regimen is an important prognostic factor and that patients with TN cancer are unlikely to benefit from metronomic regimens. To confirm that patients with $\mathrm{TN}$ 
Table IV. Clinical outcomes of patients who responded and those who did not respond to metronomic chemotherapy.

\begin{tabular}{lccc}
\hline Outcomes & Responders & Non-responders & P-value \\
\hline Response rate, $\%$ & 100 & 0 & $\mathbf{0 . 0 0 0 2}$ \\
Clinical benefit rate, $\%$ & 100 & 47.1 & $\mathbf{0 . 0 0 2 3}$ \\
Disease control rate, $\%$ & 100 & 58.8 & $\mathbf{0 . 0 0 0 0 2}$ \\
Median TTF, months & 33 & 9 & $\mathbf{0 . 0 0 0 0 0 8}$ \\
Median TTP, months & 41 & 30 & $\mathbf{0 . 0 0 0 0 2}$ \\
Median OS, months & Not reached & 40 & $\mathbf{0 . 0 0 3 3}$ \\
Median OS from MBC, months & Not reached & &
\end{tabular}

Bold print indicates statistical significance. TTF, time-to-treatment failure; TTP, time-to-progression; OS, overall survival; MBC, metastatic breast cancer.

Table V. Clinical outcomes of patients with TN cancer and those with non-TN cancer following metronomic chemotherapy.

\begin{tabular}{lccc}
\hline Outcomes & Non-TN & TN & P-value \\
\hline Response rate, $\%$ & 47.4 & 0 & $\mathbf{0 . 0 0 2 6}$ \\
Clinical benefit rate, $\%$ & 76.3 & 38.5 & $\mathbf{0 . 0 0 4 2}$ \\
Disease control rate, $\%$ & 81.6 & 53.8 & $\mathbf{0 . 0 3 6}$ \\
Median TTF, months & 16 & 7 & $\mathbf{0 . 0 0 1 4}$ \\
Median TTP, months & 24 & 8 & $\mathbf{0 . 0 0 0 2}$ \\
Median OS, months & 67 & 16 & $\mathbf{0 . 0 0 0 0 0 0 7}$ \\
Median OS from MBC, months & 108 & 20 & $\mathbf{0 . 0 0 0 0 0 0 0 2}$
\end{tabular}

Bold print indicates statistical significance. TN, triple-negative; TTF, time-to-treatment failure; TTP, time-to-progression; OS, overall survival; $\mathrm{MBC}$, metastatic breast cancer.

Table VI. Adverse events in patients who received metronomic regimens and in those who did not receive this type of regimen.

\begin{tabular}{|c|c|c|c|}
\hline Adverse events & Patients receiving MET $(\mathrm{n}=52)$ & Patients not receiving MET $(\mathrm{n}=26)$ & P-value \\
\hline Any grade, n (\%) & $19(36.5)$ & $16(61.5)$ & 0.0376 \\
\hline Grade 3/4, n (\%) & $10(19.2)$ & $11(42.4)$ & 0.0314 \\
\hline Leukopenia & 7 & 5 & \\
\hline Cystitis & $1^{\mathrm{a}}$ & - & \\
\hline Febrile neutropenia & 1 & - & \\
\hline Thrombocytopenia & $1^{\mathrm{a}}$ & - & \\
\hline Hand-foot syndrome & - & 1 & \\
\hline Heart failure & - & $1^{\mathrm{a}}$ & \\
\hline Neuropathy & - & $1^{\mathrm{a}}$ & \\
\hline Pneumonia & - & $1^{\mathrm{a}}$ & \\
\hline Proteinuria & - & 1 & \\
\hline Anorexia & - & $1^{\mathrm{a}}$ & \\
\hline
\end{tabular}

${ }^{a}$ Leading to treatment discontinuation. Bold print indicates statistical significance. MET, metronomic chemotherapy.

tumors did not benefit from metronomic regimens, baseline characteristics and clinical outcomes were compared between patients with TN tumors and those with non-TN tumors in the metronomic group. The median age was significantly higher and DFI was shorter in patients with TN tumors compared with non-TN tumors (age, 70 vs. 60 years, $\mathrm{P}=0.015$; and DFI, 63 vs. 23 months, $\mathrm{P}=0.0002$, respectively; data not shown). Other baseline characteristics did not differ between patients with TN and those with non-TN tumors. Patients with non-TN tumors (luminal-type tumors accounting for $94 \%$ of 
the non-TN tumors) were able to benefit significantly from metronomic regimens: ORR, 47.4\%; TTP, 24 months; and OS $>5$ years (Table V). By contrast, patients with TN tumors are unlikely to benefit from such regimens, as they are not expected to survive for $>2$ years.

Adverse events. To assess the toxicity of the treatment, treatment-related adverse events were compared between patients who received metronomic regimens and those who did not receive this type of regimen. The proportion of patients with severe adverse events (grades 3 and 4) was significantly lower in the metronomic group compared with that in the non-metronomic group (19.2 vs. $42.2 \%$, respectively, $\mathrm{P}=0.0314$; Table VI). In the metronomic group, 10 cases with severe adverse events included 7 cases of leukopenia, 1 case of febrile neutropenia, 1 case of hemorrhagic cystitis and 1 case of thrombocytopenia. In the non-metronomic group, 11 cases of severe adverse events included 5 cases of leukopenia, 1 case of hand-foot syndrome, 1 case of congestive heart failure, 1 case of neuropathy, 1 case of pneumonia, 1 case of proteinuria and 1 case of anorexia. Of the severe adverse events, 2 in the metronomic and 4 in the non-metronomic group led to discontinuation of treatment (Table VI). The proportion of patients with any grade of treatment-related adverse events was significantly lower in the metronomic group compared with that in the non-metronomic group (36.5 vs. $61.6 \%$, respectively; $\mathrm{P}=0.0376$ ).

\section{Discussion}

As chemotherapy agents used in the adjuvant setting, anthracyclines and taxanes are included in standard regimens, based on abundant evidence from a number of clinical trials over the past several decades, showing significant reduction in the risk of relapse or death from breast cancer (1-3). By contrast, there is no standard regimen recommended in the metastatic setting. In cases with life-threatening metastatic lesions or rapidly growing tumors, regimens that are expected to control lesions quickly, such as simultaneous administration of taxanes and anthracyclines, or taxanes in combination with either gemcitabine, capecitabine or bevacizumab, should be applied (4-9,20-23). However, a proportion of the patients exhibit disease progression during or after receiving these cytotoxic regimens, even if favorable combinations are selected. We previously demonstrated that long-term administration of one regimen was crucial for long-term survival of patients with MBC (11). To prolong the duration of treatment or TTF, chemotherapeutic regimens that are effective but less toxic are required.

Metronomic chemotherapy, which is defined as continuous or frequent treatment with low doses of anticancer agents, has been reported to result in favorable tumor responses and prolonged survival (13). We previously reported that the proportion of patients who received a metronomic regimen as the most effective regimen was two-thirds of the long-term survivors (65.3\%), who survived for $\geq 50$ months after diagnosis with $\mathrm{MBC}$, which was double that of the non-long-term survivors, who succumbed to the disease within 50 months (12). The mechanisms of inhibition of tumor growth by metronomic chemotherapy remain to be determined. Administration of low doses of cytotoxic agents is not expected to cause potent cytotoxic activity against tumor cells. However, metronomic chemotherapy has been shown in a preclinical study to elicit anti-angiogenic effects, abrogating tumor growth (14). Therefore, this treatment may continue to suppress tumor growth over a long period. In the present study, we aimed to identify a population of patients who may benefit from metronomic chemotherapy by retrospectively comparing baseline characteristics and clinical outcomes of patients with MBC who had been treated with metronomic regimens and those of patients who had been treated with non-metronomic regimens.

Among the baseline characteristics assessed in this study, median age was significantly higher, tumor grade was lower and the number of prior cytotoxic regimens was higher in the metronomic group compared with those in the non-metronomic group (Table I). The data suggest that metronomic regimens tended to be administered to patients who were elderly or had attenuated physical status, or to patients who had been heavily pretreated. As regards clinical outcome, all prognostic parameters (TTF, TTP and OS) after metronomic regimens or after the most effective of the cytotoxic regimens that patients had received, were significantly prolonged in the metronomic group compared with those in the non-metronomic group (Table II). By contrast, ORR did not differ significantly between the two groups. These data suggest that long-term treatment by one regimen is an important factor responsible for favorable prognosis, rather than temporary tumor response to different agents. In addition, the data suggest that good tumor responses to potent cytotoxic agents that have toxic side effects may not result in favorable outcomes in patients with MBC. Furthermore, we compared the clinical outcomes of patients who responded to metronomic regimens and those of patients who did not respond, in order to determine whether tumor response to metronomic regimens is required for favorable prognosis. All the prognostic factors assessed were found to be significantly better in responders compared with non-responders (Table IV). Moreover, survival time from $\mathrm{MBC}$ diagnosis was significantly prolonged in responders compared with that in non-responders, regardless of treatment line of the regimen for MBC. It was noted that almost all patients (94.4\%) who responded to metronomic regimens had hormone-sensitive luminal-type tumors (Table III). By contrast, none of patients with TN tumors responded to this type of regimen. A comparison of clinical outcomes between patients with non-TN tumors and patients with TN tumors in the metronomic group revealed that all prognostic factors, including TTF, TTP and OS, were significantly prolonged in patients with non-TN tumors compared with those in patients with TN tumors (Table V). These data suggest that metronomic regimens may be indicated for luminal-type breast cancer, but not for TN breast cancer.

As described above, not only long-term treatment with one regimen, but also a less toxic regimen, is crucial for favorable prognosis in MBC. The proportion of patients who experienced treatment-related adverse events was significantly lower in the metronomic group compared with that in the non-metronomic group (36.5 vs. 61.5\%, respectively; Table VI). Furthermore, serious grade $3 / 4$ adverse events were less frequently observed in the metronomic compared with the non-metronomic group (19.2 vs. $42.4 \%$, respectively). The results suggested that 
metronomic chemotherapy is less toxic and more tolerable in heavily pretreated patients with MBC.

In conclusion, metronomic regimens demonstrated favorable activity and minimal toxicity in patients with $\mathrm{MBC}$, regardless of prior treatment, treatment line and disease sites. In particular, this type of regimen is indicated for patients with luminal-type breast cancer and results in long-term survival, particularly in responders. However, since a metronomic regimen is unlikely to be effective in patients with TN tumors, alternative therapeutics are required for this subpopulation.

\section{Acknowledgements}

The present study was supported in part by a Grant-in-Aid for Scientific Research from the Ministry of Education, Science, Sports and Culture, Japan (grant no. 15591340).

\section{References}

1. Smith I and Chua S: Medical treatment of early breast cancer. IV: Neoadjuvant treatment. BMJ 332: 223-224, 2006.

2. Bria E, Nistico C, Cuppone F, Carlini P, Ciccarese M, Milella M, Natoli G, Terzoli E, Cognetti F and Giannarelli D: Benefit of taxanes as adjuvant chemotherapy for early breast cancer: Pooled analysis of 15,500 patients. Cancer 106: 2337-2344, 2006.

3. Buzdar AU, Singletary SE, Valero V, Booser DJ, Ibrahim NK, Rahman Z, Theriault RL, Walters R, Rivera E, Smith TL, et al: Evaluation of paclitaxel in adjuvant chemotherapy for patients with operable breast cancer: Preliminary data of a prospective randomized trial. Clin Cancer Res 8: 1073-1079, 2002.

4. O'Shaughnessy J, Miles D, Vukelja S, Moiseyenko V, Ayoub JP, Cervantes G, Fumoleau P, Jones S, Lui WY, Mauriac L, et al: Superior survival with capecitabine plus docetaxel combination therapy in anthracycline-pretreated patients with advanced breast cancer: Phase III trial results. J Clin Oncol 20: 2812-2823, 2002.

5. Albain KS, Nag SM, Calderillo-Ruiz G, Jordaan JP, Llombart AC, Pluzanska A, Rolski J, Melemed AS, Reyes-Vidal JM, Sekhon JS, et al: Gemcitabine plus paclitaxel versus paclitaxel monotherapy in patients with metastatic breast cancer and prior anthracycline treatment. J Clin Oncol 26: 3950-3957, 2009.

6. Nielsen DL, Bjerre KC, Jakobsen EH, Cold S, Stenbygaard L, Sørensen PG, Kamby C, Møller S, Jørgensen CLT and Andersson M: Gemcitabine plus docetaxel versus docetaxel in patients with predominantly human epidermal growth factor receptor 2-negative locally advanced or metastatic breast cancer: A randomized, phase III study by the Danish Breast Cancer Cooperative Group. J Clin Oncol 29: 4748-4754, 2011.

7. Miller K, Wang M, Gralow J, Dickler M, Cobleigh M, Perez EA, Shenkier T, Cella D and Davidson NE: Paclitaxel plus bevacizumab versus paclitaxel alone for metastatic breast cancer. N Engl J Med 357: 2666-2676, 2007.

8. Brufsky AM, Hurvitz S, Perez E, Swamy R, Valero V, O'Neill V and Rugo HS: RIBBON-2: A randomized, double-blind, placebo-controlled, phase III trial evaluating the efficacy and safety of bevacizumab in combination with chemotherapy for second-line treatment of human epidermal growth factor receptor 2-negative metastatic breast cancer. J Clin Oncol 29: 4286-4293, 2011.

9. Robert NJ, Diéras V, Glaspy J, Brufsky AM, Bondarenko I, Lipatov ON, Perez EA, Yardley DA, Chan SY, Zhou X, et al: RIBBON-1: Randomized, double-blind, placebo-controlled, phase III trial of chemotherapy with or without bevacizumab for first-line treatment of human epidermal growth factor receptor 2-negative, locally recurrent or metastatic breast cancer. J Clin Oncol 29: 1252-1260, 2011

10. Cortes J, O'Shaughnessy J, Loesch D, Blum JL, Vahdat LT, Petrakova K, Chollet P, Manikas A, Diéras V, Delozier T, et al: Eribulin monotherapy versus treatment of physician's choice in patients with metastatic breast cancer (EMBRACE): A phase 3 open-label randomised study. Lancet 377: 914-923, 2011.
11. Kontani K, Hashimoto SI, Murazawa C, Norimura S, Tanaka H, Ohtani M, Fujiwara-Honjo N, Date M, Houchi H and Yokomise $\mathrm{H}$ : Metronomic chemotherapy for metastatic breast cancer to prolong time to treatment failure to 12 months or more. Mol Clin Oncol 1: 225-230, 2013.

12. Kontani K, Hashimoto S, Murazawa C, Norimura S, Tanaka H, Ohtani M, Fujiwara-Honjo N, Date M, Teramoto K, Houchi H and Yokomise $\mathrm{H}$ : Factors responsible for long-term survival in metastatic breast cancer. World J Surg Oncol 12: 344, 2014.

13. Oostendorp LJ, Stalmeier PF, Donders AR, van der Graaf WT and Ottevanger PB: Efficacy and safety of palliative chemotherapy for patients with advanced breast cancer pretreated with anthracyclines and taxanes: A systematic review. Lancet Oncol 12: 1053-1061, 2011.

14. Kerbel RS and Kamen BA: The anti-angiogenic basis of metronomic chemotherapy. Nat Rev Cancer 4: 423-436, 2004.

15. Colleoni M, Rocca A, Sandri MT, Zorzino L, Masci G, Nolè F, Peruzzotti G, Robertson C, Orlando L, Cinieri S, et al: Low-dose oral methotrexate and cyclophosphamide in metastatic breast cancer: Antitumor activity and correlation with vascular endothelial growth factor levels. Ann Oncol 13: 73-80, 2002.

16. Colleoni M, Orlando L, Sanna G, Rocca A, Maisonneuve P, Peruzzotti G, Ghisini R, Sandri MT, Zorzino L, Nolè F, et al: Metronomic low-dose oral cyclophosphamide and methotrexate plus or minus thalidomide in metastatic breast cancer: Antitumor activity and biological effects. Ann Oncol 17: 232-238, 2006.

17. Wong NS, Buckman RA, Clemons M, Verma S, Dent S, Trudeau ME, Roche K, Ebos J, Kerbel R, Deboer GE, et al: Phase I/II trial of metronomic chemotherapy with daily dalteparin and cyclophosphamide, twice-weekly methotrexate, and daily prednisone as therapy for metastatic breast cancer using vascular endothelial growth factor and soluble vascular endothelial growth factor receptor levels as markers of response. J Clin Oncol 28: 723-730, 2010.

18. Eisenhauer EA, Therasse P, Bogaerts J, Schwartz LH, Sargent D, Ford R, Dancey J, Arbuck S, Gwyther S, Mooney M, et al: New response evaluation criteria in solid tumors: revised RECIST guideline (version 1.1). Eur J Cancer 45: 228-247, 2009

19. US Department of Health and Human Services. Common Terminology Criteria for Adverse Events (CTCAE), version 4.0. http://evs.nci.nih.gov/ftp1/CTCAE/CTCAE_4.03_2010-06-14_ QuickReference_8.5x11.pdf. Accessed January 14, 2014.

20. Jassem J, Pieńkowski T, Płuzańska A, Jelic S, Gorbunova V, Mrsic-Krmpotic Z, Berzins J, Nagykalnai T, Wigler N, Renard J, et al: Doxorubicin and paclitaxel versus fluorouracil, doxorubicin, and cyclophosphamide as first-line therapy for women with metastatic breast cancer: Final results of a randomized phase III multicenter trial. J Clin Oncol 19: 1707-1715, 2001

21. Bonneterre J, Dieras V, Tubiana-Hulin M, Bougnoux P, Bonneterre ME, Delozier T, Mayer F, Culine S, Dohoulou N and Bendahmane B: Phase II multicentre randomised study of docetaxel plus epirubicin vs 5-fluorouracil plus epirubicin and cyclophosphamide in metastatic breast cancer. Br J Cancer 91: 1466-1471, 2004.

22. Sledge GW, Neuberg D, Bernardo P, Ingle JN, Martino S, Rowinsky EK and Wood WC: Phase III trial of doxorubicin, paclitaxel and the combination of doxorubicin and paclitaxel as front-line chemotherapy for metastatic breast cancer: An intergroup trial (E1193). J Clin Oncol 21: 588-592, 2003.

23. Paridaens R, Biganzoli L, Bruning P, Klijn JG, Gamucci T, Houston S, Coleman R, Schachter J, Van Vreckem A, Sylvester R, et al: Paclitaxel versus doxorubicin as first-line single-agent chemotherapy for metastatic breast cancer: A European Organization for Research and Treatment of Cancer Randomized Study with cross-over. J Clin Oncol 18: 724-733, 2000 . 